\title{
Ueber binäre Formen.
}

(Von Herrn S. Gundelfinger in Tübingen.)

Herr Hermite hat im Bd. 52 dieses Journals, Seite 18 und ff. gezeigt, dass für jede gegebene binäre Form $n^{\text {ten }}$ Grades

$$
\left.f\left(x_{1}, x_{2}\right)=f^{*}\right)=\left(a_{1} x_{1}+a_{2} x_{2}\right)^{n}=a_{x}^{n}=b_{x}^{n}=\ldots
$$

eine endliche Anzahl von Covarianten existirt, durch welche alle übrigen Formen von $f$ sich rational ausdrücken lassen. Ein besonders ausgezeichnetes System solcher Covarianten ergiebt sich, indem man in $f\left(y_{1}, y_{2}\right)$ an Stelle von $y_{1}$ und $y_{2}$ neue Variable $\xi_{1}$ und $\xi_{2}$ durch die Formeln

$$
\text { (1.) } f \cdot y_{1}=\xi_{1} x_{1}-\xi_{2} \frac{1}{m} \frac{\partial f}{\partial x_{2}}, \quad f \cdot y_{2}=\xi_{1} x_{2}+\xi_{2} \frac{1}{m} \frac{\partial f}{\partial x_{1}}
$$

einführt. Man bekommt dann eine Gleichung von der Gestalt

$$
\left\{\begin{aligned}
f^{n-1} f\left(y_{1}, y_{2}\right) & =\xi_{1}^{n}+\left(\begin{array}{l}
n \\
2
\end{array}\right) f_{2} \xi_{1}^{n-2} \xi_{2}^{2}-\left(\begin{array}{l}
n \\
3
\end{array}\right) f_{3} \xi_{1}^{n-3} \xi_{2}^{3}+\cdots \\
& =F\left(\xi_{1}, \xi_{2}\right)=\left(\alpha_{1} \xi_{1}+\alpha_{2} \xi_{2}\right)^{n}=\left(\beta_{1} \xi_{1}+\beta_{2} \xi_{2}\right)^{n}=\ldots,
\end{aligned}\right.
$$

worin wir $\alpha_{1}$ und $\alpha_{2}, \beta_{1}$ und $\beta_{2}$ etc. die symbolischen Coefficienten von $F\left(\xi_{1}, \xi_{2}\right)$ bedeuten lassen und also

$$
\alpha_{1}^{n}=1, \quad \alpha_{1}^{n-1} \alpha_{2}=0, \quad \alpha_{1}^{n-2} \alpha_{2}^{2}=f_{2}, \quad \alpha_{1}^{n-3} \alpha_{2}^{3}=-f_{3} \ldots
$$

annehmen. Jede Covariante und Invariante von $f\left(y_{1}, y_{2}\right)$ ändert sich nun durch die lineare Transformation (1.) nur um eine Potenz der Substitutionsdeterminante $\frac{1}{f}$. Setzt man in der Gleichung, die dieses ausdrückt, $y_{1}=x_{1}$ und $y_{2}=x_{2}\left(\xi_{1}=f\right.$ und $\left.\xi_{2}=0\right)$, so findet man alle Formen von $f$ gleich ganzen Functionen von $f, f_{2}, f_{3}, \ldots f_{n}$, dividirt durch Potenzen von $f$.

Dieser von Herrn Hermite gemachten Entdeckung hat Herr Clebsch die weitere **) hinzugefügt, dass $f_{2}, f_{3}, \ldots f_{n}$, mit passenden Potenzen von $f$

*) Wir werden im Folgenden die Argumente $x_{1}, x_{2}$ auch bei andern Formen unterdrücken.

**) Nachrichten der Kgl. Ges. d. Wiss. zu Göttingen Jahrgang 1870, Seite 405. Wieder abgedruckt in den matbematischen Annalen von Clebsch und Neumann, Bd. III, Seite 265-267. 
multiplicirt, sich rational und ganz durch die $n$ einfacheren Formen

$$
f, \psi_{1}, \psi_{2}, \ldots \psi_{\left[\frac{n}{2}\right]}, \chi_{1}, \chi_{2}, \ldots \chi_{\left[\frac{n-1}{2}\right]}
$$

darstellen lassen müssen, wobei $\psi_{h}$ und $\chi_{h}$ durch die Gleichungen

definirt sind.

$$
\begin{aligned}
& \psi_{h}=(f, f)^{2 h}=(a b)^{2 h} a_{x}^{n-2 h} b_{x}^{n-2 h}, \\
& \chi_{h}=\left(\psi_{h}, f\right)=(a b)^{2 h}(a c) a_{x}^{n-2 h-1} b_{x}^{n-2 h} c_{x}^{n-1}
\end{aligned}
$$

Untersuchungen über die Theorie der ternären cubischen Formen, in denen ähnliche Covarianten zweier Variablen auftreten, liessen mich vermuthen, dass die $f_{i}$ selbst, ohne vorher mit Potenzen von $f$ multiplicirt werden zu müssen, rationale ganze Functionen der $\psi_{h}$ und $\chi_{h}$ seien, wie dies auch bei den durch Herrn Clebsch mitgetheilten Werthen von $f_{2}$ bis $f_{7}$ der Fall ist. In der That gelangte ich ohne Mühe zum Nachweise der Richtigkeit dieser Vermuthung, indem ich die Covarianten $(a b)^{2 h} a_{y}^{n-2 h} b_{y}^{n-2 h}$ und $(a b)^{2}(a c) a_{y}^{n-2 h-1} b_{y}^{n-2 h} c_{y}^{n-1}$ aus der Gleichung (2.) bildete und hernach $y_{1}=x_{1}, y_{2}=x_{2}$ setzte, indem ich somit dasselbe Verfahren anwandte, das oben angegeben worden, um $\psi_{h}$ und $\chi_{h}$ durch $f, f_{2}, \ldots f_{n}$ auszudrücken. Die ausfübrlichere Entwicklung dieses Gedankens, namentlich auch in seiner Anwendung auf das simultane System zweier binären Formen, bildet den Gegenstand der vorliegenden Note.

Nach (2.) hat man

$$
f^{2 n-2}(a b)^{2 h} a_{y}^{n-2 h} b_{y}^{n-2 h}=f^{2 h}(\alpha \beta)^{2 h} \alpha_{\xi}^{n-2 h} \beta_{\xi}^{n-2 h} .
$$

Durch die Annahme $y_{1}=x_{1}, y_{2}=x_{2}\left(\xi_{1}=f, \xi_{2}=0\right)$ wird die linke Seite dieser Gleichung identisch mit $f^{2 n-2} \psi_{h}$, während sich die rechte auf

$$
\begin{array}{r}
2 f^{2 n-2 h}\left(f_{2 h}+\left(\begin{array}{c}
2 h \\
2
\end{array}\right) f_{2 h-2} f_{2}-\left(\begin{array}{c}
2 h \\
3
\end{array}\right) f_{2 h-3} f_{3}+\cdots\right. \\
\left.\left.+(-1)^{h-1}\left(\begin{array}{c}
2 h \\
h-1
\end{array}\right) f_{h+1} f_{h-1}+\frac{1}{2}(-1)^{h}\left(\begin{array}{c}
2 h \\
h
\end{array}\right) f_{h}^{2}\right)^{*}\right)
\end{array}
$$

reducirt. Die letzte Relation geht also über in

$$
\left\{\begin{array}{l}
f_{2 h}=\frac{1}{2} \psi_{h} \cdot f^{2 h-2}-\left(\begin{array}{c}
2 h \\
2
\end{array}\right) f_{2 h-2} f_{2}+\left(\begin{array}{c}
2 h \\
3
\end{array}\right) f_{2 h-3} f_{3}-\cdots \\
-(-1)^{h-1}\left(\begin{array}{c}
2 h \\
h-1
\end{array}\right) f_{h+1} f_{h-1}-(-1)^{h} \frac{1}{2}\left(\begin{array}{c}
2 h \\
h
\end{array}\right) f_{h}^{2} .
\end{array}\right.
$$

Um eine Recursionsformel für $f_{2 h+1}$ zu bekommen, gehen wir von der

*) Der Coefficient von $\xi_{1}^{2 n-4 h}$ in $(\alpha \beta)^{2 h} \alpha_{\xi}^{n-2 h} \beta_{\xi}^{n-2 h}$ ist :

$$
(\alpha \beta)^{2 h} \alpha_{1}^{n-2 h} \beta_{1}^{n-2 h}=2\left(f_{2 h}+\left(\begin{array}{c}
2 h \\
2
\end{array}\right) f_{2 h-2} f_{2}-\cdots(-1)^{h-1}\left(\begin{array}{c}
2 h \\
h-1
\end{array}\right) f_{h+1} f_{h-1}+\frac{1}{2}(-1)^{h}\left(\begin{array}{c}
2 h \\
h
\end{array}\right) f_{h}^{2}\right) .
$$


evidenten Gleichung aus

$$
f^{3 n-3}(a b)^{2 h}(a c) a_{y}^{n-2 h-1} b_{y}^{n-2 h} c_{y}^{n-1}=f^{2 h+1}(\alpha \beta)^{2 h}(\alpha \gamma) \alpha_{\xi}^{n-2 h-1} \beta_{\xi}^{n-2 h} \gamma_{\xi}^{n-1} .
$$

Setzen wir in ihr $y_{1}=x_{1}, y_{2}=x_{2}$, so wird die linke Seite derselben gleich $\chi_{h} \cdot f^{3 n-3}$ und die rechte gleich

$$
\left.f^{3 n-2 h-1}\left(-2 h f_{2} f_{2 h-1}+\left(\begin{array}{c}
2 h \\
2
\end{array}\right) f_{3} f_{2 h-2}-\left(\begin{array}{c}
2 h \\
3
\end{array}\right) f_{4} f_{2 h-3}+\cdots+\left(\begin{array}{c}
2 h \\
2
\end{array}\right) f_{2 h-1} f_{2}+f_{2 h+1}\right)^{*}\right) \text {; }
$$

es ist daher :

(4.) $f_{2 h+1}=\chi_{h} \cdot f^{2 h-2}+2 h f_{2} f_{2 h-1}-\left(\begin{array}{c}2 h \\ 2\end{array}\right) f_{3} f_{2 h-2}+\left(\begin{array}{c}2 h \\ 3\end{array}\right) f_{4} f_{2 h-3}-\cdots-\left(\begin{array}{c}2 h \\ 2 h-2\end{array}\right) f_{2 h-1} f_{2}$.

Diese letzte Gleichung im Verein mit (3.) liefert das einfachste Mittel, um $f_{2}, f_{3}, \ldots f_{n}$ successive als ganze Functionen der $\psi_{h}$ und $\chi_{h}$ darzustellen.

Aehnliche Betrachtungen lassen sich auch noch anwenden, wenn zu $f$ eine andere beliebige Form $\varphi$ vom $m^{\text {ten }}$ Grade hinzutritt.

Es möge $\varphi\left(y_{1}, y_{2}\right)$ durch die Substitutionen (1.) übergehen in

$$
f^{m} \varphi\left(y_{1}, y_{2}\right)=\varphi \xi_{1}^{m}-m \varphi_{1} \xi_{1}^{m-1} \xi_{2}+\left(\begin{array}{c}
m \\
2
\end{array}\right) \varphi_{2} \xi_{1}^{m-2} \xi_{2}^{2}-\left(\begin{array}{c}
m \\
3
\end{array}\right) \varphi_{3} \xi_{1}^{m-3} \xi_{2}^{3}+\cdots=\Phi\left(\xi_{1}, \xi_{2}\right),
$$

worin also der Kürze wegen gesetzt ist:

$$
\begin{gathered}
\varphi_{1}=\frac{1}{m n}\left(\frac{\partial \varphi}{\partial x_{1}} \frac{\partial f}{\partial x_{2}}-\frac{\partial \varphi}{\partial x_{2}} \frac{\partial f}{\partial x_{1}}\right), \\
\varphi_{2}=\frac{1}{m(m-1) n^{2}}\left\{\frac{\partial^{2} \varphi}{\partial x_{1}^{2}}\left(\frac{\partial f}{\partial x_{2}}\right)^{2}-2 \frac{\partial^{2} \varphi}{\partial x_{1} \partial x_{2}} \frac{\partial f}{\partial x_{2}} \frac{\partial f}{\partial x_{1}}+\frac{\partial^{2} \varphi}{\partial x_{2}^{2}}\left(\frac{\partial f}{\partial x_{1}}\right)^{2}\right\}, \\
\varphi_{3}=\frac{1}{m(m-1)(m-2) n^{3}}\left\{\frac{\partial^{3} \varphi}{\partial x_{1}^{3}}\left(\frac{\partial f}{\partial x_{2}}\right)^{3}-3 \frac{\partial^{3} \varphi}{\partial x_{1}^{2} \partial x_{2}}\left(\frac{\partial f}{\partial x_{2}}\right)^{2} \frac{\partial f}{\partial x_{1}}+3 \frac{\partial^{3} \varphi}{\partial x_{1} \partial x_{2}^{2}} \frac{\partial f}{\partial x_{2}}\left(\frac{\partial f}{\partial x_{1}}\right)^{2}\right. \\
\left.-\frac{\partial^{3} \varphi}{\partial x_{2}^{3}}\left(\frac{\partial f}{\partial x_{1}}\right)^{3}\right\},
\end{gathered}
$$

Nach der Fundamentaleigenschaft der Covarianten ist alsdann für jede positive ganze Zahl $k \leqq n$,

$$
f^{n+m-1}\left(f\left(y_{1}, y_{2}\right), \varphi\left(y_{1}, y_{2}\right)\right)^{k}=f^{k}\left(F\left(\xi_{1}, \xi_{2}\right), \Phi\left(\xi_{1}, \xi_{2}\right)\right)^{k}
$$

*) Da $\gamma_{1}^{n}=1, \gamma_{1}^{n-1} \gamma_{2}=0$, so hat $\xi_{1}^{3 n-4 h-2}$ in $(\alpha \beta)^{2 h}(\alpha \gamma) \alpha_{\xi}^{n-2 h-1} \beta_{\xi}^{n-2 h} \gamma_{\xi}^{n-1}$ den Coefficienten

$$
\begin{gathered}
(\alpha \beta)^{2 h}(\alpha \gamma) \alpha_{1}^{n-2 h-1} \beta_{1}^{n-2 h} \gamma_{1}^{n-1}=-(\alpha \beta)^{2 h} \alpha_{1}^{n-2 h-1} \alpha_{2} \beta_{1}^{n-2 h} \\
=-2 h f_{2} f_{2 h-1}+\left(\begin{array}{c}
2 h \\
2
\end{array}\right) f_{3} f_{2 h-2}-\left(\begin{array}{c}
2 h \\
3
\end{array}\right) f_{4} f_{2 h-3}+\cdots+\left(\begin{array}{c}
2 h \\
2
\end{array}\right) f_{2 h-1} f_{2}+f_{2 h+1} .
\end{gathered}
$$

Journal für Mathematik Bd. LXXIV. Heft 1. 
Indem man in dieser Formel $y_{1}=x_{1}, y_{2}=x_{2}$ oder $\xi_{1}=f, \xi_{2}=0$ annimmt, wird die linke Seite derselben identisch mit $f^{n+m-1}(f, \varphi)^{k}$ und die rechte mit

$$
(-1)^{k} f^{m+n-k}\left(\varphi_{k}+\left(\begin{array}{c}
k \\
2
\end{array}\right) \varphi_{k-2} f_{2}-\left(\begin{array}{c}
k \\
3
\end{array}\right) \varphi_{k-3} f_{3}+\cdots+(-1)^{k} \varphi f_{k}\right)
$$

so dass man hat

$$
\varphi_{k}+\left(\begin{array}{c}
k \\
2
\end{array}\right) \varphi_{k-2} f_{2}-\left(\begin{array}{c}
k \\
3
\end{array}\right) \varphi_{k-3} f_{3}+\cdots+(-1)^{k} \varphi \cdot f_{k}=(-1)^{k} f^{k-1}(f, \varphi)^{k}
$$

Vermittelst dieser Recursionsformel kann man die $\varphi_{k}(k \leqq n)$ stufenweise rational und ganz durch $f, f_{2}, f_{3}, \ldots f_{k}$ und $(f, \varphi),(f, \varphi)^{2}, \ldots(f, \varphi)^{k}$ ausdrücken. Für $k=2$ und $k=3$ hat man z. B. mit Rücksicht auf die bekannten Werthe von $f_{2}$ und $f_{3}$ :

$$
\begin{aligned}
& \varphi_{2}=-\frac{1}{2} \varphi \cdot \psi_{1}+f(f, \varphi)^{2}, \\
& \varphi_{3}=\varphi \cdot \chi_{1}-\frac{3}{2}(\varphi, f) \psi_{1}-f^{2}(f, \varphi)^{3} .
\end{aligned}
$$

Wofern $k>n$, also etwa gleich $n+h$ ist, gestaltet sich die Recursionsformel für $\varphi_{n+h}$ nicht so einfach, wie in (5.). Wir unterdrücken daher hier die Entwicklung derselben und bemerken nur, dass man alsdann anstatt von der Gleichung (2.) von der im Grunde mit ihr identischen

$$
f^{n-1} f\left(y_{1}, y_{2}\right)\left(y_{1} \frac{1}{m} \frac{\partial f}{\partial x_{1}}+y_{2} \frac{1}{m} \frac{\partial f}{\partial x_{2}}\right)^{h}=\xi_{1}^{n+h}+\left(\begin{array}{c}
n \\
2
\end{array}\right) f_{2} \xi_{1}^{n+h-2} \xi_{2}^{2}-\left(\begin{array}{c}
n \\
3
\end{array}\right) f_{3} \xi_{1}^{n+h-3} \xi_{2}^{3} \ldots
$$

ausgehen und die $(n+h)^{\text {te }}$ Uebereinanderschiebung von

bilden muss.

$$
\varphi\left(y_{1}, y_{2}\right) \text { und } f\left(y_{1}, y_{2}\right)\left(y_{1} \frac{1}{m} \frac{\partial f}{\partial x_{1}}+y_{2} \frac{1}{m} \frac{\partial f}{\partial x_{2}}\right)^{h}
$$

Uebrigens kann man offenbar, ohne die Allgemeinheit zu beeinträchtigen, stets $m \leqq n$ voraussetzen und somit das Theorem aussprechen:

Sämmtliche Covarianten und Invarianten des simultanen Systems zweier beliebiger Formen $f$ und $\varphi$ vom $n^{\text {ten }}$ und $m^{\text {ten }}$ Grade $(m \leqq n)$ lassen sich, mit passenden Potenzen von $f$ multiplicirt, als rationale ganze Function der $m+n$ Formen

darstellen.

$$
f, \psi_{1}, \psi_{2}, \ldots \psi_{\left[\frac{n}{2}\right]} ; \quad \chi_{1}, \chi_{2}, \ldots \chi_{\left[\frac{n-1}{2}\right]}, \quad(f, \varphi),(f, \varphi)^{2}, \ldots(f, \varphi)^{m}
$$

Ausser den Anwendungen, die bereits die Herren Hermite und Clebsch von den associirten Covarianten gemacht haben, mag hier zum Schlusse noch eine 
weitere erwähnt werden. Aus der Gleichung (2.) ergiebt sich nämlich für $n=4$ und $n=3$ fast ohne alle Rechnung die Auflösung der biquadratischen und cubischen Gleichungen in der Form, wie sie von Herrn Aronhold in Bd. 52 dieses Journals Seite 95 und von mir in Bd. 2 der mathematischen Annalen von Clebsch und Neumann Seite 272 aufgestellt worden ist *).

*) Herr Clebsch, dem ich diese Auflösung mitgetheilt, schreibt mir, dass er dieselbe schon seit längerer Zeit kenne und in seinen Vorlesungen entwickelt habe.

Tübingen im Mai 1871. 